\title{
L'application du quaterne INRC sur la logique interpropositionnelle chez Piaget $\left(^{*}\right)$
}

\section{Pr .Benmissi Zoubeida Mounya ${ }^{(*)}$ Université Batna1 Algérie}

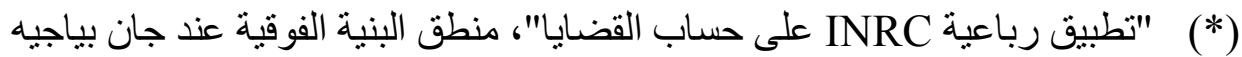

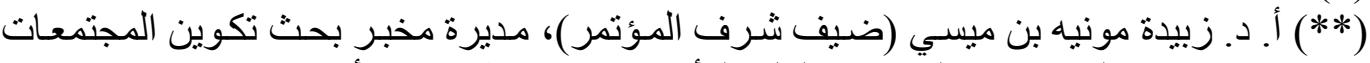

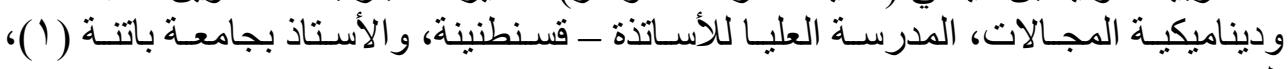
الجز ائر.
} 


\section{Introduction:}

Piaget a fixé seize connecteurs ) opérateurs(logiques qui forment seize opérations inter-propositionnelles bien définies, et il a reconnu que la théorie de la déduction ne peut être correcte sans l'existence de ces opérations qui font parties d'un système logique propositionnel, dont la base se forme de différentes transformations inter-propositionnelles qu'on peut effectuer entre les opérations, en appliquant la dérivation ainsi la réduction, celles-ci ne dépendent pas d'une opération ou d'un connecteur propositionnel précis comme le montre Scheffer, ou de deux connecteurs selon Frege, Russell, mais plutôt d'un ensemble de procédures.

Piaget a démontré que ces transformations se font en trois manières distinctes et générales: l'inverse, la réciproque et la corrélative, puis il a proposé quatre quaternes inspirés du carré d'Aristote, reliant les différentes opérations et exprimant les différentes transformations.

La question est la suivante : n'y aurait-il pas de paradoxes quand on parle du réciprocité et de l'inverse en même temps ? Quelles sont les points importants dans la conception logique de Piaget?

\section{1- Les 16 connecteurs logiques ${ }^{(1)}$ :}

Le calcul inter-propositionnel chez Piaget est basé sur un ensemble d'opérations déterminé par un certain nombre de propositions, soit une seule proposition pour les opérations uninaires ,soit deux opérations p et q qui donnent des opérations binaires, ou trois propositions pour les opérations ternaires etc..., pour les opérations binaires ,Piaget a confirmé qu'ils se resultent des combinaisons possibles de deux propositions bivalentes (c a d de deux opérations uninaires), par des "Connecteurs propositionnels", et ces conjonctions forment des nouvelles propositions dite complexes, elles sont vraie ou faux suite au valeurs de vérité de $\mathrm{p}$ et $\mathrm{q}$.

et par suite, Piaget a fixé seize connecteurs (operateurs)logiques qui forment seize opérations inter-propositionnelles définies comme suit :

(1) Jean Piaget :Traité de logique, Essai de logistique opératoire ,PUF,1949,p255. 


\begin{tabular}{|c|c|c|c|c|c|c|c|c|c|c|c|c|c|c|c|}
\hline 1 & 2 & 3 & 4 & 5 & 6 & 7 & 8 & 9 & 10 & 11 & 12 & 13 & 14 & 15 & 16 \\
\hline $\mathrm{pq}$ & - & $\mathrm{pq}$ & - & - & $\mathrm{pq}$ & $\mathrm{pq}$ & - & $\mathrm{Pq}$ & - & $p q$ & - & $\mathrm{pq}$ & - & $\mathrm{pq}$ & - \\
\hline $\mathrm{pq}$ & - & $\mathrm{pq}$ & - & $p q$ & - & - & $\mathrm{pq}^{-}$ & $p \bar{q}$ & - & - & $p q$ & $p \bar{q}$ & - & - & $\mathrm{pq}$ \\
\hline$p q$ & - & $p q$ & - & $p q$ & - & $p q$ & - & - & $\mathrm{pq}$ & - & $\mathrm{pq}$ & - & $\overline{p q}$ & $\overline{p q}$ & - \\
\hline$p q$ & - & - & $p q$ & $p q$ & - & $p q$ & & $p q$ & - & $\mathrm{pq}$ & - & & $p q$ & - & $\bar{p} \bar{q}$ \\
\hline
\end{tabular}

1- L'affirmation complète : $\mathrm{p}^{*} \mathrm{q}$

2- La négation complète: $(\cdot)$

3- La disjonction non exclusive (tri lemme :) $\mathrm{p} \vee \mathrm{q}$

4- La négation conjointe:p . q

5- L'incompatibilité :p/q

6- La conjonction : p. q

7- L'implication: $p \subset q$

8- La non implication: $\mathrm{p} \supset \mathrm{q}$

9- L'implication inverse : $q \supset p$

10- La non implication inverse $: q \supset p$

11- L'équivalence : $\mathrm{p}=\mathrm{q}$

12- La disjonction exclusive : $\mathrm{p} . \mathrm{q}$

13- L'affirmation de P : $\mathrm{p}$ [q]

14- La négation de $\mathrm{P}: \mathrm{p}[\overline{\mathrm{q}}]$

15- L'affirmation de Q : q [p]

16- La négation de $\mathrm{Q}: \mathrm{q} \overline{[\mathrm{p}]}$

\section{2- Les quatre opérations logiques:}

Après avoir exposé les seize liaisons ou opérations binaires, Piaget a étudié les opérations qui permettent de passer d'une liaison à l'autre . 
On prend arbitrairement une opération soit : $(\mathrm{p} \vee \mathrm{q})$, sa formule est:

[ (p. q) $\bar{v}(\mathrm{p} . q) \bar{v}(\mathrm{p} . \mathrm{q})]$, on peut la transformer de trois sorte différentes : l'inverse, la corrélative, la réciproque.

- l'inverse d'une opération: par exemple l'inverse de la disjonction (pvq) est la négation conjointe qui est $(\bar{p} \cdot \bar{q})$

- la réciproque de la disjonction(pvq)est

l'incompatibilité ( $p / q)$ et vice versa.

- et enfin la corrélative pour(pvq)est (p.q

Et à partir de ces transformations ,chaque opération a un inverse, uneréciproque et unecorrélative,comme l'indique le tableau suivant ${ }^{(1)}$ :

\begin{tabular}{|c|c|c|c|}
\hline $\begin{array}{l}\text { L'opération } \\
\text { directe }\end{array}$ & L'inverse & La réciproque & La corrélative \\
\hline $\mathrm{q} \vee \mathrm{p}$ & $\overline{\mathrm{p}} \cdot \overline{\mathrm{q}}$ & $\overline{\mathrm{p}} / \mathrm{q}$ & $\mathrm{q} \cdot \mathrm{p}$ \\
\hline $\mathrm{q} / \mathrm{p}$ & $\mathrm{q} \cdot \mathrm{p}$ & $\mathrm{q} \vee \mathrm{p}$ & $\overline{\mathrm{p}} \cdot \overline{\mathrm{q}}$ \\
\hline $\mathrm{q} \cdot \mathrm{p}$ & $\mathrm{q} / \mathrm{p}$ & $\overline{\mathrm{p}} \cdot \overline{\mathrm{q}}$ & $\mathrm{q} \vee \mathrm{p}$ \\
\hline$\overline{\mathrm{p}} \cdot \mathrm{q}$ & $\mathrm{q} \vee \mathrm{p}$ & $\mathrm{q} \cdot \mathrm{p}$ & $\mathrm{q} / \mathrm{p}$ \\
\hline $\mathrm{q} \supset \mathrm{p}$ & $\mathrm{p} \cdot \bar{q}$ & $\mathrm{p} \supset \mathrm{q}$ & $\overline{\mathrm{p}} \cdot \mathrm{q}$ \\
\hline $\mathrm{p} \supset \mathrm{q}$ & $\overline{\mathrm{p} . \mathrm{q}}$ & $\mathrm{q} \supset \mathrm{p}$ & $\mathrm{p} . \overline{\mathrm{q}}$ \\
\hline $\mathrm{p} . \overline{\mathrm{q}}$ & $\mathrm{q} \supset \mathrm{p}$ & $\overline{\mathrm{p}} \cdot \mathrm{q}$ & $\mathrm{p} \supset \mathrm{q}$ \\
\hline $\mathrm{p} \cdot \overline{\mathrm{q}}$ & $\mathrm{p} \supset \mathrm{q}$ & $\mathrm{p} . \overline{\mathrm{q}}$ & $\mathrm{q} \supset \mathrm{p}$ \\
\hline
\end{tabular}

(1) Ibid,p270. 


\begin{tabular}{|c|c|c|c|}
\hline $\mathrm{q}=\mathrm{p}$ & $\mathrm{qwp}$ & $\mathrm{q}=\mathrm{p}$ & Qwp \\
\hline $\mathrm{qwp}$ & $\mathrm{q}=\mathrm{p}$ & $\mathrm{qwp}$ & $\mathrm{q}=\mathrm{p}$ \\
\hline $\mathrm{q}^{*} \mathrm{p}$ & $\cdot$ & $\mathrm{q}^{*} \mathrm{p}$ & $(\cdot)$ \\
\hline$(\cdot)$ & $\mathrm{q}^{*} \mathrm{p}$ & $\cdot$ & $\mathrm{q}^{*} \mathrm{p}$ \\
\hline $\mathrm{p}[\mathrm{q}]$ & $\overline{\mathrm{p}[\mathrm{q}]}$ & $\overline{\mathrm{p}[\mathrm{q}]}$ & $\mathrm{q}[\mathrm{p}]$ \\
\hline $\bar{p}[\mathrm{q}]$ & $\mathrm{q}[\mathrm{p}]$ & $\mathrm{q}[\mathrm{p}]$ & $\bar{p}[\mathrm{q}]$ \\
\hline $\mathrm{q}[\mathrm{p}]$ & $\bar{q}[\mathrm{p}]$ & $\bar{q}[\mathrm{p}]$ & $\mathrm{p}[\mathrm{q}]$ \\
\hline $\bar{q}[\mathrm{p}]$ & $\mathrm{q}[\mathrm{p}]$ & $\mathrm{q}[\mathrm{p}]$ & $\bar{q}[\mathrm{p}]$ \\
\hline
\end{tabular}

\section{Remarques :}

1- L'opération inverse est toujours différente de l'opération directe.

2- L'opération réciproque est parfois identique à l'opération directe ou à l'opération inverse.

3- L'opération corrélative est parfois identique à l'opération directe ou à l'opération inverse.

$\mathrm{Du}$ tableau précédent, Piaget a constaté plus précisément l'existence de deux groupes, ou quaternes, composés chacun de quatre opérations distinctes, plus un quaterne dont les réciproques sont identiques entre elles et les corrélatives identiques aux inverses, enfin un dernier qui présente des corrélatives identiques entre elles et des réciproques identiques aux inverses.

\section{3- Les quatre quaternes:}

\section{-Les quaternes $A$ et $B$ :}

Ils sont représentés par huit opérations distinctes ,cad l'opération directe, l'inverse, la réciproque, la corrélative sont totalement différentes. 
On peut les exprimer par ces paires :

\begin{tabular}{|c|c|c|c|}
\hline $\begin{array}{l}\text { L'opération } \\
\text { directe }\end{array}$ & L'inverse & La réciproque & La corrélative \\
\hline $\mathrm{q} \vee \mathrm{p}$ & $\overline{\mathrm{p}} \cdot \overline{\mathrm{q}}$ & $\mathrm{p} / \mathrm{q}$ & $\mathrm{q} \cdot \mathrm{p}$ \\
\hline $\mathrm{q} \supset \mathrm{p}$ & $\mathrm{p} \cdot \mathrm{q}$ & $\mathrm{p} \supset \mathrm{q}$ & $\overline{\mathrm{p} \cdot \mathrm{q}}$ \\
\hline
\end{tabular}

Remarques :

$$
\begin{aligned}
\text { 1- Inverse } & \mathrm{pvq}=\overline{\mathrm{p}} \cdot \overline{\mathrm{q}} \\
\text { Inverse } & \mathrm{p} \supset \mathrm{q}=\mathrm{p} \cdot \mathrm{q}
\end{aligned}
$$

Et le contraire est vrai (la commutativité)

$$
\begin{array}{ll}
\text { Inverse } & \bar{p} \cdot \bar{q}=\mathrm{p} \mathrm{v} \mathrm{q} \\
\text { Inverse } & \text { p. } \bar{q}=\mathrm{q} \supset \mathrm{p}
\end{array}
$$

2- Réciproque $p v q=p / q$

Réciproque $\mathrm{p} \supset \mathrm{q}=\mathrm{q} \supset \mathrm{p}$

Réciproque $\quad \mathrm{p} / \mathrm{q}=\mathrm{p}$ v q

Réciproque $\quad \mathrm{q} \supset \mathrm{p}=\mathrm{p} \supset \mathrm{q}$

3- La corrélative = le réciproque de l'inverse ou l'inverse $\mathrm{du}$ réciproque. 


\section{le quaterne A}

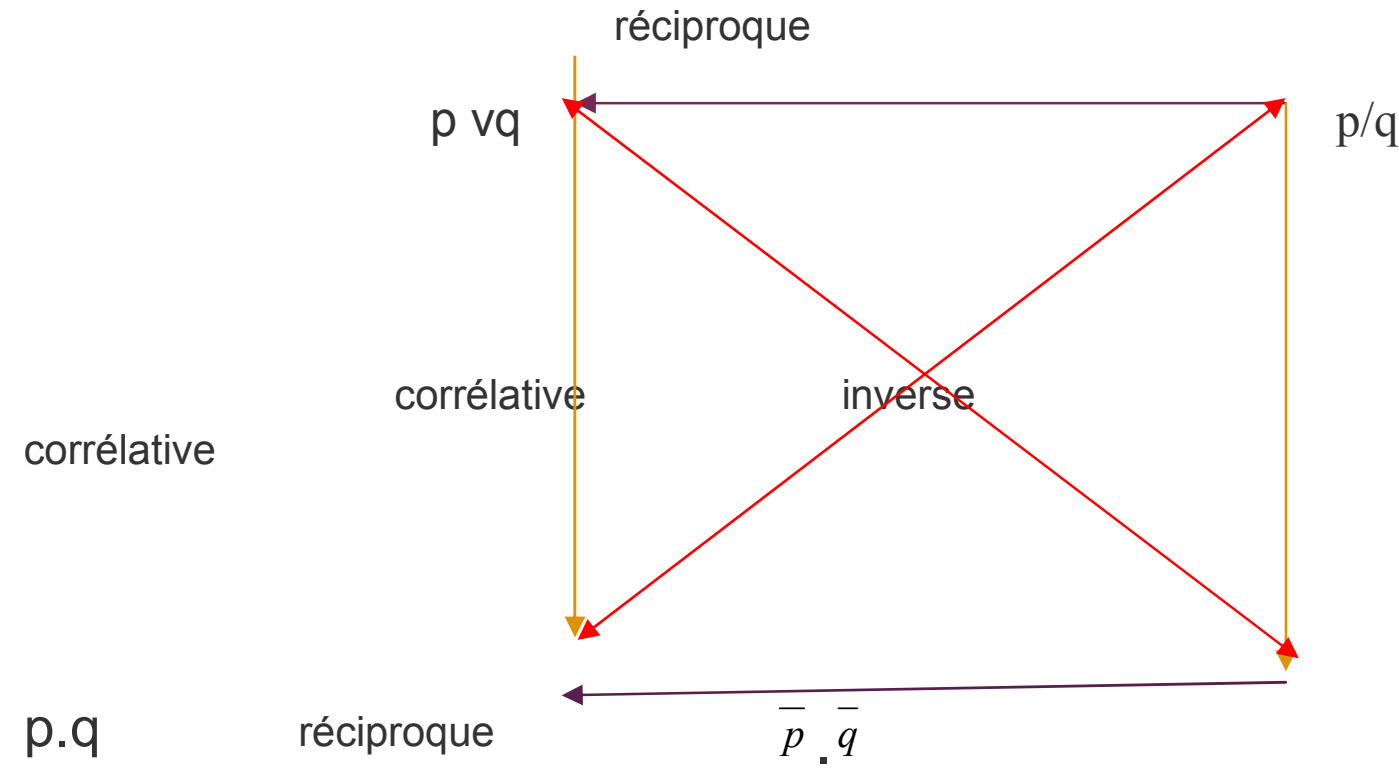




\section{le quaterne b}

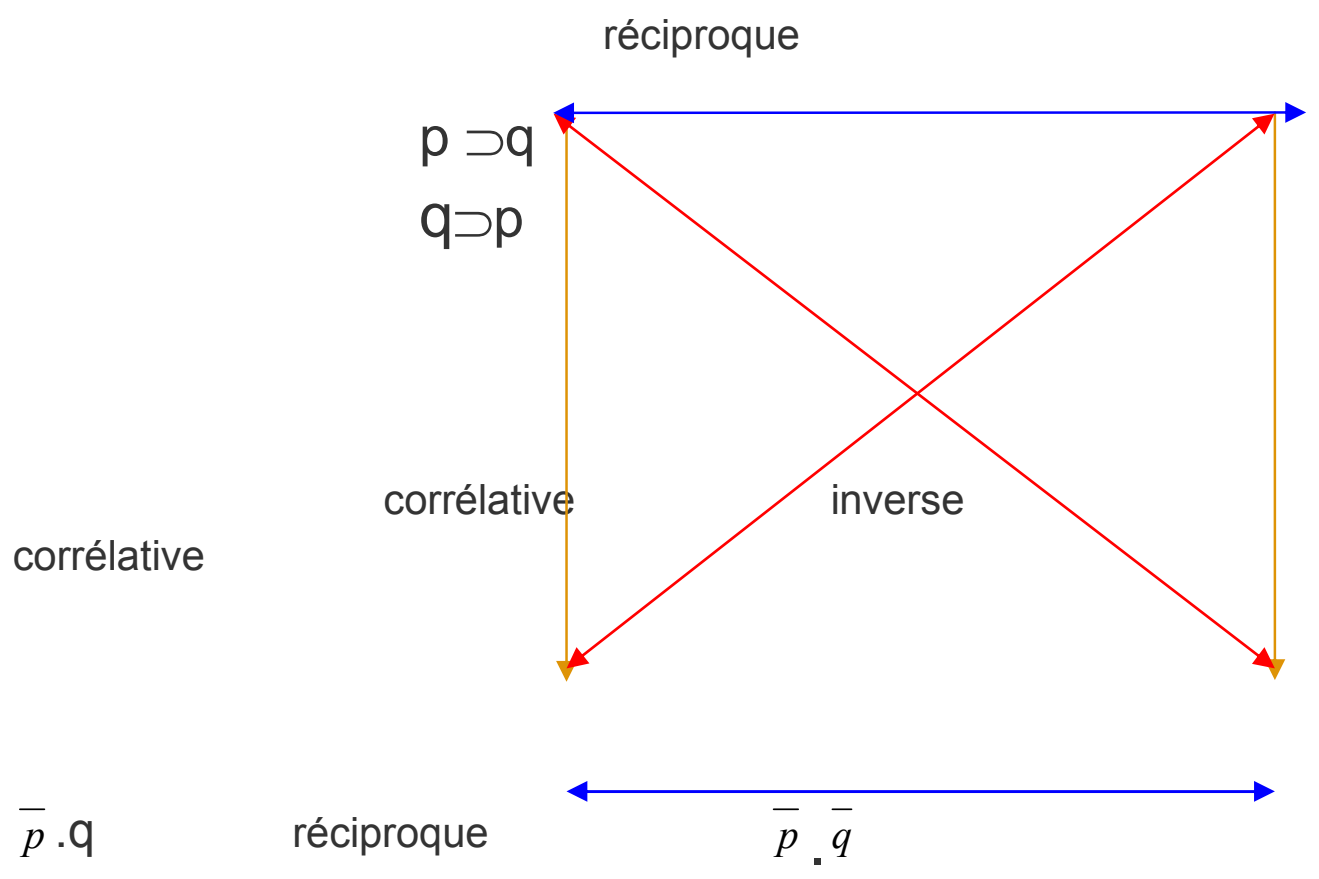

$\mathrm{Du}$ quaterne $\mathrm{A}$ on peut déduire 12 opérations logiques; quatre d'entre elles concernent l'inverse, et les quatre suivantes concernent la réciprocité et enfin les dernières sont déduites de la corrélativité .Et c'est pareil pour le quaterne B. 


\section{-Le quaterne $\mathrm{C}$ :}

où les réciproques sont identiques aux directes et les corrélatives identiques aux inverses :

\begin{tabular}{|c|c|c|c|}
\hline $\begin{array}{l}\text { L'opération } \\
\text { directe }\end{array}$ & L'inverse & La réciproque & La corrélative \\
\hline $\mathrm{p}=\mathrm{q}$ & Qwp & $\mathrm{p}=\mathrm{q}$ & $\mathrm{qwp}$ \\
\hline $\mathrm{p}^{*} \mathrm{q}$ & $\cdot$ & $\mathrm{p}^{*} \mathrm{q}$ & $(\cdot)$ \\
\hline
\end{tabular}

On exprime ces opérations par 0,2,4 paires.

réciproque

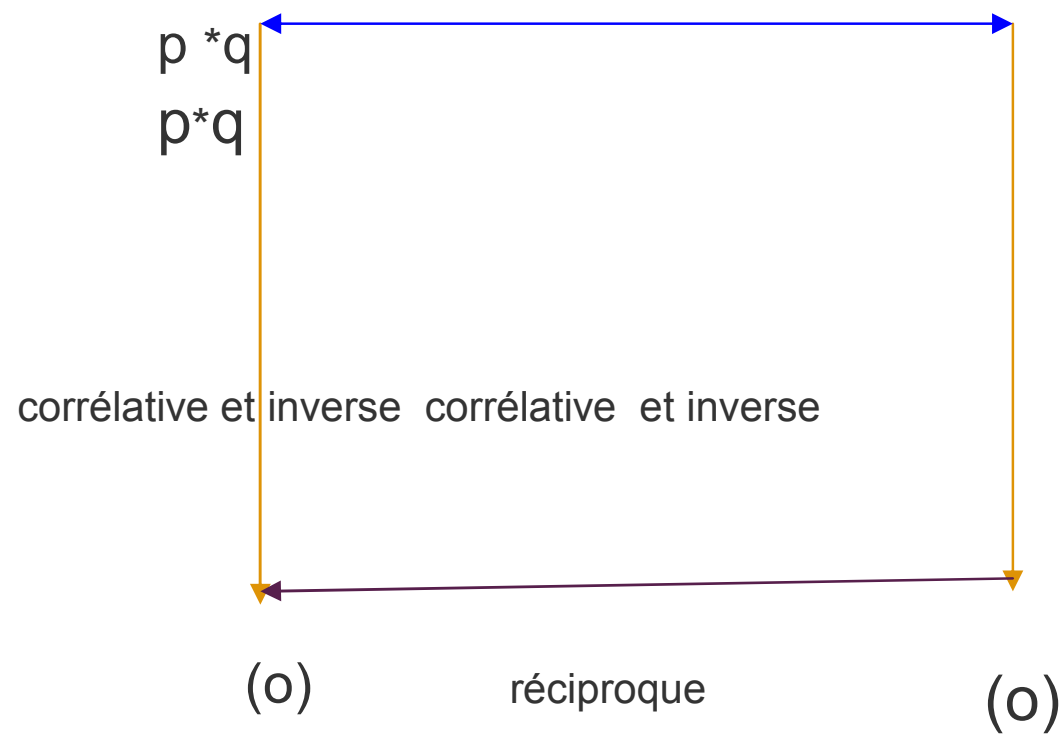




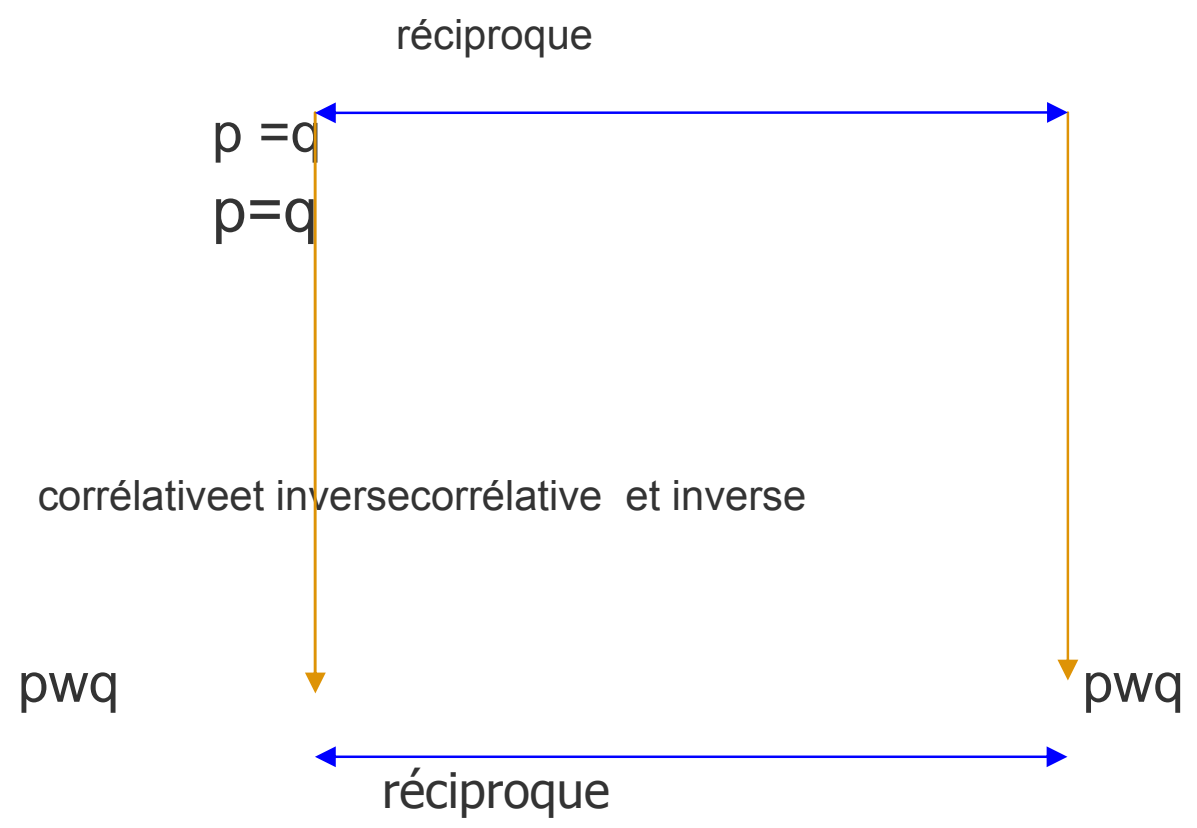

\section{le quaterne $\mathbf{D}$ :}

où on remarque l'identité entre la corrélative et l'opération directe, et entre l'inverse et la réciproque ..

\begin{tabular}{|c|c|c|c|}
\hline $\begin{array}{l}\text { L'opération } \\
\text { directe }\end{array}$ & L'inverse & La réciproque & La corrélative \\
\hline $\mathrm{P}[\mathrm{q}]$ & $\bar{p}[\mathrm{q}]$ & $\bar{p}[\mathrm{q}]$ & {$[\mathrm{p}] \mathrm{q}$} \\
\hline $\mathrm{q}[\mathrm{p}]$ & $\bar{q}[\mathrm{p}]$ & $\bar{q}[\mathrm{p}]$ & {$[\mathrm{q}] \mathrm{p}$} \\
\hline
\end{tabular}


réciprocité

correlativité $p[q]$

inverse

correlativitè $\vec{p}$ [q]

correlativité $\bar{q}$ [p]

$\stackrel{\text { inverse }}{\longleftarrow \text { réciprocité }}$

\section{4- les lois et les règles des quatre transformations:}

Après avoir étudié les mécanismes des quatre quaternes, on peut déduire

les lois de la logique inter-propositionnelle: A) La loi du double réversibilité( ${ }^{(1)}$ :

Et parmi ses théories l'inverse: l'inverse du réciproque d'une opération est identique au réciproque de son inverse. B) la réciprocité :

- Réciproque de l'inverse=inverse de l'inverse

- Réciproque de l'inverse= inverse ,ssi opération directe $=$ réciproque

- Réciproque de $\mathrm{A}=$ inverse de la corrélative de $\mathrm{A}$

- Si réciproque de A est vraie $\Rightarrow$ corrélative fausse

- Si corrélative est vraie $\Rightarrow$ réciproque est fausse

C)si la réciprocité change la valeur de p sans q ou vice versa $\Rightarrow$ réciproque $=$ inverse...

(1) Piaget: La réversibilité de la pense et les opérations logiques, Bulletin de la société française de la philosophie, 1950,p137. 


\section{5-Le groupe commutatif I N R C :}

Après avoir démontré une série de théorèmes par rapport à ces différents quaternes d'opérations, Piaget expose le théorème central de sa logique des propositions, l'existence d'un groupe de transformations entre les quatre opérations agissant sur les seize opérations base de la logique bivalente.

\section{C'est le groupe INRC.}

Les quatre transformations forment un groupe commutatif ${ }^{(1)}$ :

Si on met :

Inverse $=\mathrm{N}$

Corrélative $=\mathrm{C}$

Réciproque $=\mathrm{R}$

Opération directe $=\mathrm{I}$

Remarques :

- La répétition de chaque opération nous ramène à l'opération directe ou identique :

Corrélative de la corrélative $=1$

Inverse de l'inverse $=1$

$(N N)=1,(C C)=1,(R R)=1(\mathrm{I})$

$(N)^{2}=1,(C)^{2}=1,(R)^{2}=1(\mathrm{I})$

- corrélative du réciproque $=$ réciproque du corrélative $=$ inverse

$\mathrm{CR}=\mathrm{RC}=\mathrm{N}$

-réciproque de l'inverse $=$ corrélative $=$-inverse du réciproque

$\mathrm{RN}=\mathrm{NR}=\mathrm{C}$

-Inverse du corrélative $=$ corrélative inverse $=$ réciproque

$\mathrm{NC}=\mathrm{CN}=\mathrm{R}$

(1) J.Piaget : Le groupe des transformations de la logique des propositions bivalentes, Archives des sciences, Genève, 1949,p179. 
Le groupe INRC ${ }^{(1)}$ :

$\mathrm{CR}=\mathrm{RC}=\mathrm{N}$

$\mathrm{RN}=\mathrm{NR}=\mathrm{C}$

$\mathrm{NC}=\mathrm{CN}=\mathrm{R}$

$$
\begin{array}{cccc}
\mathrm{I} & \mathrm{R} & \mathrm{N} & \mathrm{C} \\
\mathrm{R} & 1 & \mathrm{C} & \mathrm{N} \\
\mathrm{N} & \mathrm{C} & 1 & \mathrm{R} \\
\mathrm{C} & \mathrm{N} & \mathrm{R} & 1
\end{array}
$$

Démonstration:est ce qu'il est commutatif?

a- La loi de composition interne, par exemple:

$(\mathrm{RN})=\mathrm{C}$ et $\mathrm{NN}=\mathrm{RR}=\mathrm{CC}=1$

b-l 'associativité : $\mathrm{N}(\mathrm{CR})=\mathrm{C}(\mathrm{RN})$

du tableau précédent, on peut démontrer que:

$(\mathrm{CR})=\mathrm{N}, \mathrm{NN}=1$

$(\mathrm{NR})=\mathrm{C}, \mathrm{CC}=1$

Donc,,$(\mathrm{N}) \mathrm{N}=(\mathrm{C}) \mathrm{C}=1$

c) élément neutre:

L'opération 1 joue le rôle d'élément neutre, ce qui est indiqué dans la ligne verticale et horizontale du carré.

d - l'élément inversé:

Comme le montre le tableau, chaque élément estl' inverse de lui-même.

e-commutativité :

Le tableau montre que: ... $\mathrm{CN}=\mathrm{NC} ; \mathrm{NR}=\mathrm{RN}$ donc le groupe INRC est commutatif.

(1) J. Piaget : La réversibilité de la pense et les opérations logiques,op.ci,p181. 


\section{6- Opérations ternaires et INRC:}

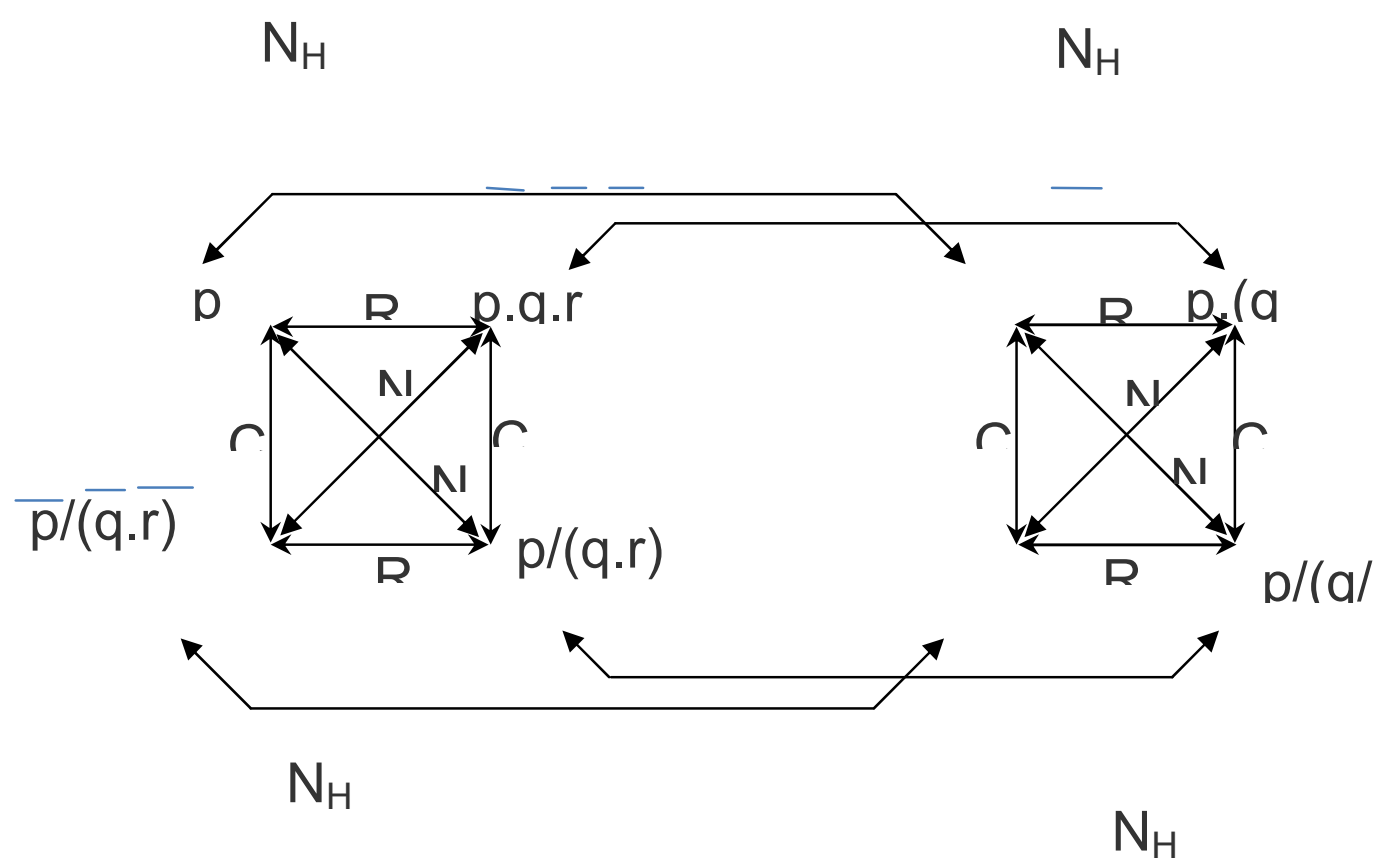

Cette figure est l'un des nombreux exemples résultant de l'ajout de $N_{H}$ à l'INRC, et la même chose pour les transformations $R_{H}, C_{H}$ et d'autres transformations qui ont aidé à la construction du système logique des opérations ternaires de la logique bivalente des propositions ${ }^{(1)}$.

L'avantage du calcul des opérations ternaires est qu'il est riche en questions et analyses logiques, contrairement aux opérations binaires, car il est basé sur les 256 opérations ternaires, d'autant plus les réduire aux opérations double binaires ou mono binaires, puis des transformations qui peuvent être effectués grâce à la structure INRC, ainsi l'identification d'un ensemble de familles d'opérations , chaque famille est caractérisée par la combinaison de transformations inconnues dans les opérations binaires, et encore la transition possible d'une famille à l'autre ...

(1) Voir : Piaget: Essai sur les transformation des opérations logiques les 256 opérations ternaires,PUF,Paris, 1952. 
Ainsi, la logique de calcul des opérations ternaires est plus large que la logique de calcul des opérations binaires logiquement et psychologiquement..

Et bien la logique du calcul des propositions est développée grâce à Piaget, en découvrant le groupe INRC qui a été reconnu par de nombreux logiciens, aussi la mise en place d'un système du calcul des propositions soit d'opérations binaires ou ternaires .

Tout logicien pourra appliquer le système de calcul de des propositions sur quatre propositions en indiquant leur nombre, caractéristiques ou même les applications et les transformations les plus importantes qu'ils peuvent subir, tout en notant dans le cas de quatre propositions, nous obtenons 65536 opérations cad 164. 


\section{Conclusion :}

L'enfant dans le stade des opérations concrètes reste lié dans sa pensée à la réalité, c'est-à-dire que son intelligence nela transcende pas; il est incapable de comprendre des concepts abstraits, des hypothèses ou des principes, donc la pensée est la perception d'un fait possible, tandis que la pensée abstraite La pensée abstraite va au-delà de la réalité pour se retrouver dans le monde des concepts abstraits, qui place de nouvelles possibilités intellectuelles pour les adolescents.

Cette pensée commence vers l'âge 11-12 ans, où l'individu peut penser d'une manière déductive ((Hypothético déductives) ;l'enfant s'appuie sur des hypothèses et des concepts qu'on appelle opérations formelles, et non pas sur des thèmes ressentis.

Cela se traduit par la réalisation de l'équilibre dépendant de la réalisation du principe de réversibilité du réciproque et de l'inverse ,représentés dans le groupe I N R C, à travers la composition des 16 opérations logiques déduites des deux propositions par l'enfant.

Le groupe des quatre transformations propre à la logique interpropositionnellede l'adolescent (inversion, réciprocité, inversion de la réciproque ou réciproque de l'inverse et transformation identique) montre... comment deux formes de la réversibilité opératoire finissent par se coordonner en un système unique. Du point de vue psychologique, le groupe INRC désigne l'une des structures qui agit au sein de la pensée formelle en lui donnant une puissance opératoire accrue par rapport aux structures caractérisant l'intelligence représentative concrète.

Piaget a pu combiner entre l'inverse et la réciprocité sans qu'il y'aura des paradoxes mais plutôt ça a enrichie la logique bivalente. 
